\title{
Electromagnetic field quantization in a linear polarizable and magnetizable medium
}

\author{
F. Kheirandish ${ }^{1}$ and M. Amooshahi ${ }^{1} \dagger$ \\ 1 Department of Physics, University of Isfahan, \\ Hezar Jarib Ave., Isfahan, Iran.
}

October 3, 2018

\begin{abstract}
By modeling a linear polarizable and magnetizable medium (magnetodielectric) with two quantum fields, namely $\mathrm{E}$ and $\mathrm{M}$, electromagnetic field is quantized in such a medium consistently and systematically. A Hamiltonian is proposed from which, using the Heisenberg equations, Maxwell and constitutive equations of the medium are obtained. For a homogeneous medium, the equation of motion of the quantum vector potential, $\vec{A}$, is derived and solved analytically. Two coupling functions which describe the electromagnetic properties of the medium are introduced. Four examples are considered showing the features and the applicability of the model to both absorptive and nonabsorptive magneto-dielectrics.

Keywords: Field quantization, Magneto-dielectric, Electric and Magnetic Susceptibility, Noise density, Coupling function

PACS number: 12.20.Ds
\end{abstract}

\section{Introduction}

In a homogeneous and nondispersive medium, the photon is associated with only the transverse part of the electromagnetic field. In contrast in an in-

*fardin_kh@phys.ui.ac.ir

†amooshahi@sci.ui.ac.ir 
homogeneous nondispersive medium, the transverse and the longitudinal degrees of freedom are coupled. In this case the quantization of the electromagnetic field can be accomplished by employing a generalized gauge that is, $\vec{\nabla} \cdot(\varepsilon(\vec{r}) \vec{A})=0$, where $\varepsilon(\vec{r})$, is the space dependent dielectric function $[1,2]$. Using the gauge $\sum_{i, j=1}^{3} \frac{\partial}{\partial x_{i}}\left(\varepsilon_{i j}(\vec{r}) \vec{A}_{j}\right)=0$, the generalization of this quantization to the case of an anisotropic nondispersive medium is straightforward $[3]$.

The quantization in a dispersive and absorptive dielectric represents one of the most interesting problems in quantum optics, since it gives a rigorous test of our understanding of the interaction of light with matter. The dissipative nature of a medium is an immediate consequence of its dispersive character and vice versa according to the Kramers-Kronig relations. This means that the validity of the electromagnetic field quantization in a nondissipative but dispersive media, is restricted to a range of frequencies for which the imaginary part of the dielectric function is negligible. Otherwise, there will be inconsistencies in electromagnetic field quantization process.

In the scheme of Lenac for dispersive and nonabsorptive dielectric media, by starting from fundamental equations of motion, the medium is described by a dielectric function $\varepsilon(\vec{r}, \omega)$ without any restriction on its spatial behavior [4]. In this scheme, it is assumed that there are no losses in the system, so the dielectric function is real for the whole space. The procedure is based on an expansion of the total field in terms of the coupled eigen-modes, orthogonality relations are derived and equal-time commutation relations are discussed.

Huttner and Barnett have presented a canonical quantization for electromagnetic field inside an absorptive dielectric [5]. In their model, the medium is represented by a collection of interacting matter fields and the absorptive character of the medium is described by interaction of the matter fields with a reservoir containing a continuum of Klein-Gordon fields. In this model, eigen-operators for the coupled systems are calculated and electromagnetic field is expressed in terms of these operators. Also the dielectric function is derived and is shown to satisfy the Kramers-Kronig relations.

Gruner and Welsch presented a quantization method of the radiation field inside a dispersive and absorptive linear dielectric starting from the phenomenological Maxwell equations, where the properties of the dielectric are described by a permitivity consistent with the Kramers-Kronig relations [6]. An expansion of the field operators is performed which is based on the Green function of the classical Maxwell equations and preserves the equal- 
time canonical commutation relations.

Suttorp and Wubs in the framework of the damped polarization model, have quantized the electromagnetic field in an absorptive medium with spatial dependence of its parameters [7]. They have solved the equations of motion of the dielectric polarization and the electromagnetic field by means of the Laplace transformation for both positive and negative times. The operators that diagonalize the Hamiltonian are found as linear combinations of canonical variables with coefficients depending on the electric susceptibility and the dielectric Green function. Also the time dependence of the electromagnetic field and the dielectric polarization are determined.

The macroscopic description of a quantum damped harmonic oscillator with frequency $\omega_{0}$ is represented in terms of the Langevin equation $[8,9,10]$ :

$$
\ddot{\vec{x}}+\int_{0}^{\infty} d t^{\prime} \mu\left(t-t^{\prime}\right) \dot{\vec{x}}\left(t^{\prime}\right)-\omega_{0}^{2} \int_{0}^{\infty} d t^{\prime} \nu\left(t-t^{\prime}\right) \vec{x}\left(t^{\prime}\right)=F_{N}(t) .
$$

The coupling with the heat bath in the microscopic theory corresponds to two types of forces in the macroscopic equation of motion of a damped harmonic oscillator. The forces of the first type are obtained from some memory functions $\mu$ and $\nu$. The second type force is the noise force $\vec{F}_{N}(t)$. These two types of forces have a fluctuation-dissipation connection and both are required for a consistent description of a dissipative quantum system.

Matloob has quantized the macroscopic electromagnetic field in a linear isotropic permeable dielectric medium by quantizing the Langevin equation and associating a damped quantum harmonic oscillator with each mode of the radiation field [10]. There are some other approaches to quantizing the electromagnetic field and interested reader is referred to [11-21].

In this paper by modeling a polarizable and magnetizable medium with two massless quantum fields, namely "E and M quantum fields", we come to a completely systematic and consistent method for quantizing electromagnetic field in such a medium. We propose a general method in which the medium is included in quantization process. In fact, the coupling of electromagnetic field with the medium in a microscopic level, is modeled macroscopically by replacing the medium with "E and $\mathrm{M}$ quantum fields ". The underlying mechanism of interaction between electromagnetic field and the medium in microscopic theory may be much more complicated than that implied by this model which simply replace the medium with a collection of harmonic oscillators. The present model is a generalization of Caldeira-Leggett model where for dissipative quantum systems, they model the environment by a 
collection of harmonic oscillators $[22,23]$. In the Caldeira-Leggett model, the environment's Hamiltonian is

$$
H_{B}=\sum_{n}\left[\frac{p_{n}^{2}}{2 m_{n}}+\frac{1}{2} m_{n} \omega_{n}^{2} x_{n}^{2}\right]
$$

where $m_{n}, x_{n}, p_{n}$ and $\omega_{n}$ are mass, position, momentum and frequency of $n$th oscillator respectively. It should be noted that the properties of the environment may in some cases be determined on the basis of a microscopic model which is not necessarily the Caldeira-Leggett model. As an example we mention an Ohmic resistor which as a linear electric element should be well described by a Hamiltonian of the form (2). On the other hand the underlying mechanism leading to dissipation in a resistor may be more complicated than that implied by the model of a collection of harmonic oscillators.

The main feature of the present approach is that " $\mathrm{E}$ and $\mathrm{M}$ quantum fields" are to describe the electric and magnetic properties of the medium macroscopically. It is clear (at least in the present work, based on the results), that this assumption is an effective one which can be compared with the phenomenological approaches to the problem.

In the Green function method and schemes applied in references [6], 10], [13, 14, 24] the noise current and polarization densities are due to interaction of electromagnetic field with the medium. Since the kind of this interaction is not defined, the explicit forms of the noise current and polarization densities are not known. It is clear that the dissipative character of the medium depends on electric and magnetic susceptibilities. Since the presence of the noise quantum fields are necessary for quantum dissipative systems, there should be a dependence between the strengths of the noise densities and the strengths of electric and magnetic susceptibilities. Specially, when the medium becomes a non dissipative one, i.e., when the imaginary part of the Fourier transform of dielectric function tends to zero, the strengths of the noise densities should clearly tend to zero. In the Green function method and schemes applied in 6] 10, [13, 14, 24, the relation between the strengths of the noise densities and the strengths of electric and magnetic susceptibilities is not clear. Therefore it is not clear how these noises tend to zero when the medium becomes a non dissipative medium.

The constitutive equations of the medium which relate electric and magnetic polarization densities to electric and magnetic fields respectively, should be treated as a consequence of interaction between electromagnetic field and the medium. But in the mentioned methods, the constitutive equations can 
not be derived from Heisenberg equations of motion. In the present approach, the coupling of electromagnetic field with the medium is known and explicit forms of noise densities are given. Therefore the relation between the strengths of noise densities and the strengths of electric and magnetic susceptibilities is clear. Also, in this approach, the constitutive equations can be derived from the Heisenberg equations of motion. The key role is played by what we have introduced as coupling functions, which couple the electromagnetic field to "E and M quantum fields". The electric and magnetic susceptibilities are defined in terms of the coupling functions. Also, the noise polarization densities are described in terms of the coupling functions and the creation and annihilation operators of the "E and M quantum fields". The coupling functions are common factors in the noise densities and the electric and magnetic susceptibilities. It can be shown that for a non dissipative medium, the noise densities vanish as expected.

In the damped polarization model the polarizability property of the dielectric, is described by a quantum field $\mathrm{Y}$ and the dissipative property of the medium is described by introducing a heat bath which is independent of the field Y. The heat bath is a continuum of Klein - Gordon fields with a continuous frequency range. The heat bath interacts with the medium in a suitable way. In this model, the magnetic property of the medium is not included $[5,7]$.

In the present approach, the magnetic property of the medium is included and the polarizability and the dissipative properties of the medium are described only in terms of a single quantum field (E quantum field). Also, the electric susceptibility is defined in terms of a coupling function which couples the "E quantum field" to electromagnetic field. Finally, the electric polarization field of the medium is defined in terms of the coupling function and the creation and annihilation operators of the "E quantum field".

\section{Quantum dynamics}

Quantum electrodynamics in a linear polarizable and magnetizable medium can be accomplished by modeling the medium with two independent quantum fields which interact with the electromagnetic field. One of these quantum fields namely " E quantum field", describes the polarizability character of the medium and interacts with the displacement field $\vec{D}$ through a minimal cou- 
pling term. The other quantum field, namely " M quantum field", describes magnetizability character of the medium and interacts with magnetic field through a dipole interaction term. The Heisenberg equations for eletromagnetic field (system) and " E and M quantum fields" (environment), lead to both Maxwell and constitutive equations. The constitutive equations relate the electric and magnetic polarization densities to the macroscopic electric and magnetic fields, respectively.

The vector potential of the electromagnetic field in Coulomb gauge can be expanded in terms of the plane waves as

$$
\vec{A}(\vec{r}, t)=\int d^{3} \vec{k} \sum_{\lambda=1}^{2} \sqrt{\frac{\hbar}{2(2 \pi)^{3} \varepsilon_{0} \omega_{\vec{k}}}}\left[a_{\vec{k} \lambda}(t) e^{i \vec{k} \cdot \vec{r}}+a_{\vec{k} \lambda}^{\dagger}(t) e^{-i \vec{k} \cdot \vec{r}}\right] \vec{e}_{\vec{k} \lambda}
$$

where $\omega_{\vec{k}}=c|\vec{k}|$ and $\varepsilon_{0}$ is the permitivity of the vacuum. The unit vectors $\vec{e}_{\vec{k} \lambda}, \quad(\lambda=1,2)$ are polarization vectors and satisfy

$$
\begin{aligned}
\vec{e}_{\vec{k} \lambda} \cdot \vec{e}_{\vec{k} \lambda^{\prime}} & =\delta_{\lambda \lambda^{\prime}}, \\
\vec{e}_{\vec{k} \lambda} \cdot \vec{k} & =0 .
\end{aligned}
$$

These recent relations, guarantee that the vector potential (3), satisfies the Coulomb gauge $\vec{\nabla} \cdot \vec{A}(\vec{r}, t)=0$.

Operators $a_{\vec{k} \lambda}(t)$ and $a_{\vec{k} \lambda}^{\dagger}(t)$ are annihilation and creation operators of electromagnetic field and satisfy the following equal-time commutation rules

$$
\left[a_{\vec{k} \lambda}(t), a_{\overrightarrow{k^{\prime} \lambda^{\prime}}}^{\dagger}(t)\right]=\delta\left(\vec{k}-\overrightarrow{k^{\prime}}\right) \delta_{\lambda \lambda^{\prime}}
$$

The conjugate canonical momentum density of the electromagnetic field $\vec{\pi}_{F}(\vec{r}, t)$ and also the displacement vector operator $\vec{D}(\vec{r}, t)$ are by definition

$$
\vec{\pi}_{F}(\vec{r}, t)=-\vec{D}(\vec{r}, t)=i \varepsilon_{0} \int d^{3} \vec{k} \sum_{\lambda=1}^{2} \sqrt{\frac{\hbar \omega_{\vec{k}}}{2(2 \pi)^{3} \varepsilon_{0}}}\left[a_{\vec{k} \lambda}^{\dagger}(t) e^{-i \vec{k} \cdot \vec{r}}-a_{\vec{k} \lambda}(t) e^{i \vec{k} \cdot \vec{r}}\right] \vec{e}_{\vec{k} \lambda} .
$$

From this definition it is obvious that $\nabla \cdot \vec{D}=0$, which is the Gauss law in the absence of external charges. The commutation relations (5) lead to the commutation relations between the components of the vector potential $\vec{A}$ and the displacement vector operator $\vec{D}$ as

$$
\left[A_{l}(\vec{r}, t),-D_{j}\left(\overrightarrow{r^{\prime}}, t\right)\right]=\left[A_{l}(\vec{r}, t), \pi_{j}\left(\overrightarrow{r^{\prime}}, t\right)\right]=\imath \hbar \delta_{l j}^{\perp}\left(\vec{r}-\overrightarrow{r^{\prime}}\right)
$$


where $\delta_{l j}^{\perp}\left(\vec{r}-\overrightarrow{r^{\prime}}\right)=\frac{1}{(2 \pi)^{3}} \int d^{3} \vec{k} e^{i \vec{k} \cdot\left(\vec{r}-\overrightarrow{r^{\prime}}\right)}\left(\delta_{l j}-\frac{k_{l} k_{j}}{|\vec{k}|^{2}}\right)$, is the transverse delta function with the following properties:

1. Let us define the transverse and longitudinal components of an arbitrary vector field $\vec{F}(\vec{r}, t)$, as

$$
\begin{aligned}
& \vec{F}^{\perp}(\vec{r}, t)=\vec{F}(\vec{r}, t)-\int d^{3} r^{\prime} \nabla^{\prime} \cdot \vec{F}\left(\overrightarrow{r^{\prime}}, t\right) \vec{\nabla} G\left(\vec{r}, \overrightarrow{r^{\prime}}\right), \\
& \vec{F}^{\|}(\vec{r}, t)=\int d^{3} r^{\prime} \nabla^{\prime} \cdot \vec{F}\left(\overrightarrow{r^{\prime}}, t\right) \vec{\nabla} G\left(\vec{r}, \overrightarrow{r^{\prime}}\right),
\end{aligned}
$$

respectively. Wherein $G\left(\vec{r}, \overrightarrow{r^{\prime}}\right)$ is the Green function

$$
G\left(\vec{r}, \overrightarrow{r^{\prime}}\right)=-\frac{1}{4 \pi\left|\vec{r}-\overrightarrow{r^{\prime}}\right|}
$$

Then as the first property, one can easily show that

$$
F_{i}^{\perp}(\vec{r}, t)=\sum_{j=1}^{3} \int d^{3} r^{\prime} \delta_{i j}^{\perp}\left(\vec{r}-\overrightarrow{r^{\prime}}\right) F_{j}\left(\overrightarrow{r^{\prime}}\right) .
$$

2. The second property is the transversality of $\delta_{l j}^{\perp}\left(\vec{r}-\overrightarrow{r^{\prime}}\right)$

$$
\sum_{l=1}^{3} \frac{\partial}{\partial x_{l}} \delta_{l j}^{\perp}\left(\vec{r}-\overrightarrow{r^{\prime}}\right)=0, \quad j=1,2,3 .
$$

The Hamiltonian of the electromagnetic field inside a magneto-dielectric medium, can be written as

$$
\begin{aligned}
H_{F}(t) & =\int d^{3} r\left[\frac{\vec{D}^{2}(\vec{r}, t)}{2 \varepsilon_{0}}+\frac{(\nabla \times \vec{A})^{2}(\vec{r}, t)}{2 \mu_{0}}\right], \\
& =\sum_{\lambda=1}^{2} \int d^{3} \vec{k} \hbar \omega_{\vec{k}} a_{\vec{k} \lambda}^{\dagger}(t) a_{\vec{k} \lambda}(t),
\end{aligned}
$$

where $\mu_{0}$ is the magnetic permitivity of the vacuum and we have applied the normal ordering to operators $a_{\vec{k} \lambda}^{\dagger}(t)$ and $a_{\vec{k} \lambda}(t)$.

Now as mentioned, we model the medium by two quantum fields, $E$ and $M$, which describe the electric and magnetic properties of the medium 
macroscopically. Therefore, the Hamiltonian of the medium can be written as

$$
\begin{aligned}
H_{d} & =H_{e}(t)+H_{m}(t), \\
H_{e}(t) & =\sum_{\nu=1}^{3} \int d^{3} \vec{q} \int d^{3} \vec{k} \hbar \omega_{\vec{k}} d_{\nu}^{\dagger}(\vec{k}, \vec{q}, t) d_{\nu}(\vec{k}, \vec{q}, t), \\
H_{m}(t) & =\sum_{\nu=1}^{3} \int d^{3} \vec{q} \int d^{3} \vec{k} \hbar \omega_{\vec{k}} b_{\nu}^{\dagger}(\vec{k}, \vec{q}, t) b_{\nu}(\vec{k}, \vec{q}, t) .
\end{aligned}
$$

where $\omega_{\vec{k}}$ is the dispersion relation of the medium and $H_{e}$ and $H_{m}$ are the Hamiltonians of the "E and M quantum fields", respectively. The operators $d_{\nu}(\vec{k}, \vec{q}, t), d_{\nu}^{\dagger}(\vec{k}, \vec{q}, t), b_{\nu}(\vec{k}, \vec{q}, t)$ and $b_{\nu}^{\dagger}(\vec{k}, \vec{q}, t)$, are annihilation and creation operators of the "E and M quantum fields", respectively. We impose the following equal-time commutation relations on these operators

$$
\begin{aligned}
& {\left[d_{\nu}(\vec{k}, \vec{q}, t), d_{\nu^{\prime}}^{\dagger}\left(\overrightarrow{k^{\prime}}, \overrightarrow{q^{\prime}}, t\right)\right]=\delta_{\nu \nu^{\prime}} \delta\left(\vec{k}-\vec{k}^{\prime}\right) \delta\left(\vec{q}-\vec{q}^{\prime}\right),} \\
& {\left[b_{\nu}(\vec{k}, \vec{q}, t), b_{\nu^{\prime}}^{\dagger}\left(\overrightarrow{k^{\prime}}, \overrightarrow{q^{\prime}}, t\right)\right]=\delta_{\nu \nu^{\prime}} \delta\left(\vec{k}-\vec{k}^{\prime}\right) \delta\left(\vec{q}-\vec{q}^{\prime}\right) .}
\end{aligned}
$$

Now let us define the electric polarization density operator of the medium as

$$
\vec{P}(\vec{r}, t)=\sum_{\nu=1}^{3} \int \frac{d^{3} \vec{q}}{\sqrt{(2 \pi)^{3}}} \int d^{3} \vec{k}\left[f\left(\omega_{\vec{k}}, \vec{r}\right) d_{\nu}(\vec{k}, \vec{q}, t) e^{i \vec{q} \cdot \vec{r}}+H . C .\right] \vec{v}_{\nu}(\vec{q}),
$$

where for convenience H.C. stands for Hermitian Conjugation and

$$
\begin{array}{ll}
\vec{v}_{\nu}(\vec{q})=\vec{e}_{\nu \vec{q}}, \quad \text { for } \quad \nu=1,2, \\
\vec{v}_{3}(\vec{q})=\hat{q}=\frac{\vec{q}}{|\vec{q}|}, \quad \text { for } \quad \nu=3 .
\end{array}
$$

In a similar manner we define the magnetic polarization density operator of the medium as

$$
\begin{array}{lll}
\vec{M}(\vec{r}, t)=i \sum_{\nu=1}^{3} \int \frac{d^{3} \vec{q}}{\sqrt{(2 \pi)^{3}}} \int d^{3} \vec{k}\left[g\left(\omega_{\vec{k}}, \vec{r}\right) b_{\nu}(\vec{k}, \vec{q}, t) e^{i \vec{q} \cdot \vec{r}}-H . C .\right] \vec{s}_{\nu}(\vec{q}), & \\
\vec{s}_{\nu}(\vec{q})=\hat{q} \times \vec{e}_{\nu \vec{q}}, & \text { for } & \nu=1,2 \\
\vec{s}_{3}(\vec{q})=\hat{q}, & \text { for } & \nu=3 .
\end{array}
$$


In the polarization densities (16) and (18), the functions $f\left(\omega_{\vec{k}}, \vec{r}\right)$ and $g\left(\omega_{\vec{k}}, \vec{r}\right)$ are the coupling functions between the electromagnetic field and " $\mathrm{E}$ and $\mathrm{M}$ quantum fields". The coupling functions are position dependent (independent) for a inhomogeneous (homogeneous) magneto-dielectrics.

The polarization densities $\vec{P}$ and $\vec{M}$, are defined on the basis of the following physical assumptions:

1- These densities should be Hermitian operators.

2- For a linear magneto-dielectric medium, $\vec{P}$ and $\vec{M}$, should be a linear combination of creation and annihilation operators of the medium. For a non-linear medium, $\vec{P}$ and $\vec{M}$, may not have a linear expansion in terms of the creation and annihilation operators of the medium.

3- Polarization densities should depend on the macroscopic properties of the medium.

Macroscopic properties of the medium will be reflected in electric and magnetic susceptibilities. It is clear from relations (16) and (18) that the polarization densities $\vec{P}$ and $\vec{M}$ depend on coupling functions $f\left(\omega_{k}, \vec{r}\right), g\left(\omega_{k}, \vec{r}\right)$ and dispersion relation $\left(\omega_{\vec{k}}\right)$ of the medium. In the following we show that electric and magnetic susceptibilities are dependent on the coupling functions and dispersion relation.

Now let us propose the total Hamiltonian as

$$
\begin{aligned}
\tilde{H}(t) & =\int d^{3} r\left\{\frac{[\vec{D}(\vec{r}, t)-\vec{P}(\vec{r}, t)]^{2}}{2 \varepsilon_{0}}+\frac{(\nabla \times \vec{A})^{2}(\vec{r}, t)}{2 \mu_{0}}-\nabla \times \vec{A}(\vec{r}, t) \cdot \vec{M}(\vec{r}, t)\right\} \\
& +H_{e}+H_{m} .
\end{aligned}
$$

Using (17) and (11), we can obtain the Heisenberg equations for $\vec{A}$ and $\vec{D}$

$$
\begin{gathered}
\frac{\partial \vec{A}(\vec{r}, t)}{\partial t}=\frac{\imath}{\hbar}[\tilde{H}, \vec{A}(\vec{r}, t)]=-\frac{\vec{D}(\vec{r}, t)-\vec{P}^{\perp}(\vec{r}, t)}{\varepsilon_{0}}, \\
\frac{\partial \vec{D}(\vec{r}, t)}{\partial t}=\frac{\imath}{\hbar}[\tilde{H}, \vec{D}(\vec{r}, t)]=\frac{\nabla \times \nabla \times \vec{A}(\vec{r}, t)}{\mu_{0}}-\nabla \times \vec{M}^{\perp}(\vec{r}, t),
\end{gathered}
$$

where $\vec{P}^{\perp}$ and $\vec{M}^{\perp}$ are transverse components of $\vec{P}$ and $\vec{M}$ respectively. The transverse electric field $\vec{E}^{\perp}$, magnetic induction $\vec{B}$ and magnetic field $\vec{H}$ are

$$
\vec{E}^{\perp}=-\frac{\partial \vec{A}}{\partial t}, \quad \vec{B}=\nabla \times \vec{A}, \quad \vec{H}=\frac{\vec{B}}{\mu_{0}}-\vec{M},
$$


so (201) and (21) can be rewritten as

$$
\begin{gathered}
\vec{D}=\varepsilon_{0} \vec{E}^{\perp}+\vec{P}^{\perp}, \\
\frac{\partial \vec{D}}{\partial t}=\nabla \times \vec{H}^{\perp} .
\end{gathered}
$$

In absence of an external charge density, we have $\vec{D}^{\|}=\varepsilon_{0} \vec{E}^{\|}+\vec{P} \|=0$, and we can define the longitudinal component of the electric field as $\vec{E}^{\|}=-\frac{\vec{P} \|}{\varepsilon_{0}}$. Combining (20) and (21), we find

$$
-\nabla^{2} \vec{A}+\frac{1}{c^{2}} \frac{\partial^{2} \vec{A}}{\partial t^{2}}=\mu_{0} \frac{\partial \vec{P}^{\perp}}{\partial t}+\mu_{0} \nabla \times \vec{M}^{\perp} .
$$

By using the commutation relations (15), the Heisenberg equations for operators $d_{\nu}(\vec{k}, \vec{q}, t)$ and $b_{\nu}(\vec{k}, \vec{q}, t)$ can be obtained as

$$
\begin{aligned}
\dot{d}_{\nu}(\vec{k}, \vec{q}, t) & =\frac{\imath}{\hbar}\left[\tilde{H}, d_{\nu}(\vec{k}, \vec{q}, t)\right] \\
& =-\imath \omega_{\vec{k}} d_{\nu}(\vec{k}, \vec{q}, t)+\frac{\imath}{\hbar \sqrt{(2 \pi)^{3}}} \int d^{3} r^{\prime} f^{*}\left(\omega_{\vec{k}}, \overrightarrow{r^{\prime}}\right) \vec{E}\left(\overrightarrow{r^{\prime}}, t\right) e^{-i \vec{q} \cdot \overrightarrow{r^{\prime}}} \cdot \overrightarrow{v_{\nu}}(\vec{q}),
\end{aligned}
$$

and

$$
\begin{aligned}
\dot{b}_{\nu}(\vec{k}, \vec{q}, t) & =\frac{\imath}{\hbar}\left[\tilde{H}, b_{\nu}(\vec{k}, \vec{q}, t)\right] \\
& =-\imath \omega_{\vec{k}} b_{\nu}(\vec{k}, \vec{q}, t)+\frac{1}{\hbar \sqrt{(2 \pi)^{3}}} \int d^{3} r^{\prime} g^{*}\left(\omega_{\vec{k}}, \overrightarrow{r^{\prime}}\right) e^{-i \vec{q} \cdot \overrightarrow{r^{\prime}}} \vec{B}\left(\overrightarrow{r^{\prime}}, t\right) \cdot \vec{s}_{\nu}(\vec{q})
\end{aligned}
$$

respectively. These equations have the following formal solutions

$$
\begin{aligned}
& d_{\nu}(\vec{k}, \vec{q}, t)=d_{\nu}(\vec{k}, \vec{q}, 0) e^{-\imath \omega_{\vec{k}} t}+ \\
& \frac{\imath}{\hbar \sqrt{(2 \pi)^{3}}} \int_{0}^{t} d t^{\prime} e^{-\imath \omega_{\vec{k}}\left(t-t^{\prime}\right)} \int d^{3} r^{\prime} f^{*}\left(\omega_{\vec{k}}, \overrightarrow{r^{\prime}}\right) e^{-i \vec{q} \cdot \overrightarrow{r^{\prime}}} \vec{E}\left(\overrightarrow{r^{\prime}}, t^{\prime}\right) \cdot \vec{v}_{\nu}(\vec{q}), \\
& b_{\nu}(\vec{k}, \vec{q}, t)=b_{\nu}(\vec{k}, \vec{q}, 0) e^{-\imath \omega_{\vec{k}} t}+ \\
& \frac{1}{\hbar \sqrt{(2 \pi)^{3}}} \int_{0}^{t} d t^{\prime} e^{-\imath \omega_{\vec{k}}\left(t-t^{\prime}\right)} \int d^{3} r^{\prime} g^{*}\left(\omega_{\vec{k}}, \overrightarrow{r^{\prime}}\right) e^{-i \vec{q} \cdot \overrightarrow{r^{\prime}}} \vec{B}\left(\overrightarrow{r^{\prime}}, t^{\prime}\right) \cdot \vec{s}_{\nu}(\vec{q}) .
\end{aligned}
$$


By substituting (28) in (16), we find the polarization operator

$$
\vec{P}(\vec{r}, t)=\vec{P}_{N}(\vec{r}, t)+\varepsilon_{0} \int_{0}^{|t|} d t^{\prime} \chi_{e}\left(\vec{r},|t|-t^{\prime}\right) \vec{E}\left(\vec{r}, \pm t^{\prime}\right)
$$

where $\vec{E}=-\frac{\partial \vec{A}}{\partial t}-\frac{\vec{P} \|}{\varepsilon_{0}}$ is the total electric field. The upper (lower) sign, corresponds to $t>0(t<0)$ respectively.

The memory function

$$
\begin{array}{ll}
\chi_{e}(\vec{r}, t)=\frac{8 \pi}{\hbar \varepsilon_{0}} \int_{0}^{\infty} d|\vec{k}||\vec{k}|^{2}\left|f\left(\omega_{\vec{k}}, \vec{r}\right)\right|^{2} \sin \omega_{\vec{k}} t & t>0 \\
\chi_{e}(\vec{r}, t)=0 & t \leq 0
\end{array}
$$

is called the electric susceptibility of the magneto-dielectric which is defined in terms of dispersion relation $\omega_{\vec{k}}$ and the coupling function $f\left(\omega_{\vec{k}}, \vec{r}\right)$. The operator $\vec{P}_{N}(\vec{r}, t)$ in (30) is the noise electric polarization density

$\vec{P}_{N}(\vec{r}, t)=\sum_{\nu=1}^{3} \int \frac{d^{3} \vec{q}}{\sqrt{(2 \pi)^{3}}} \int d^{3} \vec{k}\left[f\left(\omega_{\vec{k}}, \vec{r}\right) d_{\nu}(\vec{k}, \vec{q}, 0) e^{-\imath \omega_{\vec{k}} t+i \vec{q} \cdot \vec{r}}+H . C.\right] \vec{v}_{\nu}(\vec{q})$.

By using the equation (31), we can obtain the following important relations in frequency domain

$$
\begin{gathered}
\operatorname{Im}\left[\underline{\chi}_{e}(\vec{r}, \omega)\right]=\frac{4 \pi^{2}}{3 \hbar \varepsilon_{0}} \frac{d|\vec{k}|^{3}}{d \omega}|f(\vec{r}, \omega)|^{2} \\
\operatorname{Re}\left[\underline{\chi}_{e}(\vec{r}, \omega)\right]=\frac{8 \pi}{\hbar \varepsilon_{0}} \int_{0}^{\infty} d|\vec{k}||\vec{k}|^{2}\left|f\left(\omega_{\vec{k}}, \vec{r}\right)\right|^{2} \frac{\omega_{\vec{k}}}{\omega_{\vec{k}}^{2}-\omega^{2}}
\end{gathered}
$$

where

$$
\underline{\chi}_{e}(\vec{r}, \omega)=\int_{0}^{\infty} d t \chi_{e}(\vec{r}, t) e^{\imath \omega t}
$$

A feature of the present approach is its flexibility to choosing an appropriate dispersion relation $\omega(|\vec{k}|)$, such that $\frac{d \omega}{d|\vec{k}|}>0$. When a dispersion relation is given, then knowing the susceptibility, we can obtain the corresponding coupling function from (33) easily. The sign of the left hand side of the relation (33) should be positive because it is the imaginary part of the electric susceptibility in the frequency domain and this imaginary part is also connected to losses in the medium, so it is necessarily positive, otherwise $\underline{\chi}_{e}(\vec{r}, \omega)$ 
should be discarded, as it would be unphysical. Therefore for consistency one must choose a dispersion relation which is a strictly increasing function of $|\vec{k}|$. Here we have chosen the simplest one, i.e. a linear dispersion relation. It is remarkable to note that for a given susceptibility $\underline{\chi}_{e}(\vec{r}, \omega)$, by using (15) and the definition of $\vec{P}_{N}$ in (32), one can show that for any choice of $\omega(|\vec{k}|)$ and $f(\omega, \vec{r})$ satisfying (33) , the following commutation relations

$$
\left[\underline{P}_{N i}(\vec{r}, \omega), \underline{P}_{N j}\left(\overrightarrow{r^{\prime}}, \omega^{\prime}\right)\right]=\frac{\hbar \varepsilon_{0}}{\pi} \operatorname{Im}\left[\underline{\chi}_{e}(\vec{r}, \omega)\right] \delta_{i j} \delta\left(\vec{r}-\overrightarrow{r^{\prime}}\right) \delta\left(\omega-\omega^{\prime}\right)
$$

between the Fourier transforms of the components of the noise polarization density do not change. These commutation relations are identical with those in reference 13 and lead to the correct commutation relations of electromagnetic field operators. So various choices of $\omega(|\vec{k}|)$ and $f(\omega, \vec{r})$ restricted by (33), do not affect the commutation relations (36) and also the commutation relations between electromagnetic field operators. This means that there are many models with the same electric and magnetic properties which can be taken as an environment with different dispersion relations. In this point of view $\omega(|\vec{k}|)$ and $f(\omega, \vec{r})$ are two free parameters of our model up to the relation (33). As was mentioned, we choose the simplest dispersion relation $\omega(|k|)=c|\vec{k}|$, where c, velocity of light, as the proportionality coefficient, is just for simplifying the calculations. It is clear from equation (33) that other choices of the dispersion relation, just lead to a redefinition of the coupling function $f(\omega, \vec{r})$ and also more difficult mathematical expressions.

For the choice $\omega(|k|)=c|\vec{k}|$, the definition of the electric susceptibility in (31), becomes

$$
\begin{aligned}
\chi_{e}(\vec{r}, t) & =\frac{8 \pi}{\hbar c^{3} \varepsilon_{0}} \int_{0}^{\infty} d \omega_{\vec{k}} \omega_{\vec{k}}^{2}\left|f\left(\omega_{\vec{k}}, \vec{r}\right)\right|^{2} \sin \omega_{\vec{k}} t, & & t>0 \\
\chi_{e}(\vec{r}, t) & =0, & & t \leq 0
\end{aligned}
$$

For a definite $\chi_{e}(\vec{r}, t)$, which is zero for $t \leq 0$, we can obtain the corresponding coupling function $f\left(\omega_{\vec{k}}, \vec{r}\right)$, in terms of $\chi_{e}(\vec{r}, t)$, as

$$
\begin{aligned}
\left|f\left(\omega_{\vec{k}}, \vec{r}\right)\right|^{2} & =\frac{\hbar c^{3} \varepsilon_{0}}{4 \pi^{2} \omega_{\vec{k}}^{2}} \int_{0}^{\infty} d t \chi_{e}(\vec{r}, t) \sin \omega_{\vec{k}} t, & \omega_{\vec{k}}>0 \\
\left|f\left(\omega_{\vec{k}}, \vec{r}\right)\right|^{2} & =0, & \omega_{\vec{k}}=0 .
\end{aligned}
$$


Similarly, by substituting (29) in (18), we obtain the following expression for the magnetic polarization density $\vec{M}(\vec{r}, t)$,

$$
\vec{M}(\vec{r}, t)=\vec{M}_{N}(\vec{r}, t)+\frac{1}{\mu_{0}} \int_{0}^{|t|} d t^{\prime} \chi_{m}\left(\vec{r},|t|-t^{\prime}\right) \vec{B}\left(\vec{r}, \pm t^{\prime}\right)
$$

where $\chi_{m}$ is the magnetic susceptibility of the magneto-dielectric

$$
\begin{array}{lll}
\chi_{m}(\vec{r}, t)=\frac{8 \pi \mu_{0}}{\hbar c^{3}} \int_{0}^{\infty} d \omega_{\vec{k}} \omega_{\vec{k}}^{2}\left|g\left(\omega_{\vec{k}}, \vec{r}\right)\right|^{2} \sin \omega_{\vec{k}} t, & & t>0, \\
\chi_{m}(\vec{r}, t)=0, & & t \leq 0 .
\end{array}
$$

If we are given a definite $\chi_{m}(\vec{r}, t)$, which is zero for $t \leq 0$, then we can obtain the corresponding coupling function $g\left(\omega_{\vec{k}}, \vec{r}\right)$ in terms of $\chi_{m}(\vec{r}, t)$ as

$$
\begin{array}{rlrl}
\left|g\left(\omega_{\vec{k}}, \vec{r}\right)\right|^{2} & =\frac{\hbar c^{3}}{4 \pi^{2} \mu_{0} \omega_{\vec{k}}^{2}} \int_{0}^{\infty} d t \chi_{m}(\vec{r}, t) \sin \omega_{\vec{k}} t, & \omega_{\vec{k}}>0, \\
\left|g\left(\omega_{\vec{k}}, \vec{r}\right)\right|^{2}=0, & \omega_{\vec{k}}=0 .
\end{array}
$$

The operator $M_{N}(\vec{r}, t)$, is the noise magnetic polarization density

$$
\begin{aligned}
& \vec{M}_{N}(\vec{r}, t)= \\
& i \sum_{\nu=1}^{3} \iint \frac{d^{3} \vec{q}}{\sqrt{(2 \pi)^{3}}} d^{3} \vec{k}\left[g\left(\omega_{\vec{k}}, \vec{r}\right) b_{\nu}(\vec{k}, \vec{q}, 0) e^{-\imath \omega_{\vec{k}} t+i \vec{q} \cdot \vec{r}}-H . C .\right] \vec{s}_{\nu}(\vec{q}) .
\end{aligned}
$$

It is remarkable to note that the constitutive equations (23), (30) and (39) together with Maxwell equations are obtained directly from the Heisenberg equations applied to the electromagnetic field and the quantum fields $E$ and $M$. The explicit forms of the noise polarization densities are given by (32) and (42). The coupling functions $f$ and $g$, are common factors in the noise densities $\vec{P}_{N}$ and $\vec{M}_{N}$ and the susceptibilities $\chi_{e}, \chi_{m}$. So it is clear that the strengths of the noise fields are dependent on the strengths of the susceptibilities $\chi_{e}$ and $\chi_{m}$, which describe the dissipative character of a magnetodielectric medium.

For a homogeneous medium, the coupling functions $f\left(\omega_{\vec{k}}, \vec{r}\right)$ and $g\left(\omega_{\vec{k}}, \vec{r}\right)$, are position independent. In this case, from (37) and (40), we deduce that 
$\chi_{e}$ and $\chi_{m}$, are also position independent. Substituting (30) and (39) in the wave equation (25), we find

$$
\begin{aligned}
& -\nabla^{2} \vec{A}+\frac{1}{c^{2}} \frac{\partial^{2} \vec{A}}{\partial t^{2}} \pm \frac{1}{c^{2}} \frac{\partial}{\partial t} \int_{0}^{|t|} d t^{\prime} \chi_{e}\left(|t|-t^{\prime}\right) \frac{\partial \vec{A}}{\partial t^{\prime}}\left(\vec{r}, \pm t^{\prime}\right)- \\
& \nabla \times \int_{0}^{|t|} d t^{\prime} \chi_{m}\left(|t|-t^{\prime}\right) \nabla \times \vec{A}\left(\vec{r}, \pm t^{\prime}\right)=\mu_{0} \frac{\partial \vec{P}_{N}^{\perp}}{\partial t}(\vec{r}, t)+\mu_{0} \nabla \times \vec{M}_{N}(\vec{r}, t),
\end{aligned}
$$

where $c^{2}=\frac{1}{\varepsilon_{0} \mu_{0}}$ and the upper (lower) sign corresponds to $t>0(t<0)$ respectively.

The equation (43) is the Langevin-Schrödinger equation [8] for vector the potential $\vec{A}$, wherein, the explicit form of the noise current density is known.

The quantum Langevin equation can be considered as the basis of the macroscopic description of a quantum particle coupled to an environment or a heat bath [8, 9].

\section{Solution of the Heisenberg equations}

In this section, we solve the Heisenberg equations for the vector potential. Let us denote the Fourier transform of the vector potential $\vec{A}(\vec{r}, t)$ by $\underline{\vec{A}}(\vec{q}, t)$, so

$$
\vec{A}(\vec{r}, t)=\frac{1}{\sqrt{(2 \pi)^{3}}} \int d^{3} \vec{q} \underline{\vec{A}}(\vec{q}, t) e^{i \vec{q} \cdot \vec{r}}
$$

from (3), it is clear that

$$
\underline{\vec{A}}(\vec{q}, t)=\sum_{\lambda=1}^{2} \sqrt{\frac{\hbar}{2 \varepsilon_{0} \omega_{\vec{q}}}}\left[a_{\vec{q} \lambda}(t) \vec{e}_{\vec{q} \lambda}+a_{-\vec{q} \lambda}^{\dagger}(t) \vec{e}_{-\vec{q} \lambda}\right] .
$$

The wave equation (43) can be written in terms of $\underline{\vec{A}}(\vec{q}, t)$ as

$$
\begin{aligned}
& \underline{\vec{A}}+\omega_{\vec{q}}^{2} \underline{\vec{A}} \pm \frac{\partial}{\partial t} \int_{0}^{|t|} d t^{\prime} \chi_{e}\left(|t|-t^{\prime}\right) \underline{\dot{\vec{A}}}\left(\vec{q}, \pm t^{\prime}\right)-\omega_{\vec{q}}^{2} \int_{0}^{|t|} d t^{\prime} \chi_{m}\left(|t|-t^{\prime}\right) \underline{\vec{A}}\left(\vec{q}, \pm t^{\prime}\right) \\
= & -\frac{\imath}{\varepsilon_{0}} \sum_{\lambda=1}^{2} \int \frac{d^{3} \vec{k}}{\sqrt{(2 \pi)^{3}}}\left[\omega_{\vec{k}} f\left(\omega_{\vec{k}}\right) d_{\lambda}(\vec{k}, \vec{q}, 0) e^{-\imath \omega_{\vec{k}} t} \vec{e}_{\vec{q} \lambda}-\omega_{\vec{k}} f^{*}\left(\omega_{\vec{k}}\right) d_{\lambda}^{\dagger}(\vec{k},-\vec{q}, 0) e^{\imath \omega_{\vec{k}} t} \vec{e}_{-\vec{q} \lambda}\right]
\end{aligned}
$$




$$
+\omega_{\vec{q}} \sqrt{\frac{\mu_{0}}{\varepsilon_{0}}} \sum_{\lambda=1}^{2} \int \frac{d^{3} \vec{k}}{\sqrt{(2 \pi)^{3}}}\left[g\left(\omega_{\vec{k}}\right) b_{\lambda}(\vec{k}, \vec{q}, 0) e^{-\imath \omega_{\vec{k}} t} \vec{e}_{\vec{q} \lambda}+g^{*}\left(\omega_{\vec{k}}\right) b_{\lambda}^{\dagger}(\vec{k},-\vec{q}, 0) e^{\imath \omega_{\vec{k}} t} \vec{e}_{-\vec{q} \lambda}\right]
$$

where $\omega_{\vec{q}}=c|\vec{q}|$.

This equation can be solved using the Laplace transformation method. For any time dependent operator $g(t)$, the forward and backward Laplace transformations are by definition

$$
g_{f}(s)=\int_{0}^{\infty} d t g(t) e^{-s t}
$$

and

$$
g_{b}(s)=\int_{0}^{\infty} d t g(-t) e^{-s t}
$$

respectively. Let $\tilde{\chi}_{e}(s)$ and $\tilde{\chi}_{m}(s)$ be the Laplace transformations of $\chi_{e}(t)$ and $\chi_{m}(t)$ respectively. Then $\underline{\vec{A}}_{f}(\vec{q}, s)$ and $\underline{\vec{A}}_{b}(\vec{q}, s)$, i.e., the forward and backward Laplace transformation of $\vec{A}(\vec{q}, t)$, can be obtained in terms of $\tilde{\chi}_{e}(s)$ and $\tilde{\chi}_{m}(s)$ as

$$
\begin{aligned}
& \underline{\vec{A}}_{f, b}(\vec{q}, s)=\frac{s+s \tilde{\chi}_{e}(s)}{s^{2}+\omega_{\vec{q}}^{2}+s^{2} \tilde{\chi}_{e}(s)-\omega_{\vec{q}}^{2} \tilde{\chi}_{m}(s)} \underline{\vec{A}}(\vec{q}, 0) \\
& \pm \frac{1}{s^{2}+\omega_{\vec{q}}^{2}+s^{2} \tilde{\chi}_{e}(s)-\omega_{\vec{q}}^{2} \tilde{\chi}_{m}(s)} \underline{\dot{\vec{A}}}(\vec{q}, 0) \\
& -\frac{\imath}{\varepsilon_{0}} \sum_{\lambda=1}^{2} \int d^{3} \vec{k}\left\{\frac{\omega_{\vec{k}} f\left(\omega_{\vec{k}}\right) d_{\lambda}(\vec{k}, \vec{q}, 0)}{\left(s \pm \imath \omega_{\vec{k}}\right)\left[s^{2}+\omega_{\vec{q}}^{2}+s^{2} \tilde{\chi}_{e}(s)-\omega_{\vec{q}}^{2} \tilde{\chi}_{m}(s)\right]} \vec{e}_{\vec{q} \lambda}\right. \\
& \left.-\frac{\omega_{\vec{k}} f^{*}\left(\omega_{\vec{k}}\right) d_{\lambda}^{\dagger}(\vec{k},-\vec{q}, 0)}{\left(s \mp \imath \omega_{\vec{k}}\right)\left[s^{2}+\omega_{\vec{q}}^{2}+s^{2} \tilde{\chi}_{e}(s)-\omega_{\vec{q}}^{2} \tilde{\chi}_{m}(s)\right]} \vec{e}_{-\vec{q} \lambda}\right\} \\
& +\sqrt{\frac{\mu_{0}}{\varepsilon_{0}}} \omega_{\vec{q}} \sum_{\lambda=1}^{2} \int d^{3} \vec{k}\left\{\frac{g\left(\omega_{\vec{k}}\right) b_{\lambda}(\vec{k}, \vec{q}, 0)}{\left(s \pm \imath \omega_{\vec{k}}\right)\left[s^{2}+\omega_{\vec{q}}^{2}+s^{2} \tilde{\chi}_{e}(s)-\omega_{\vec{q}}^{2} \tilde{\chi}_{m}(s)\right]} \vec{e}_{\vec{q} \lambda}\right. \\
& \left.+\frac{g^{*}\left(\omega_{\vec{k}}\right) b_{\lambda}^{\dagger}(\vec{k},-\vec{q}, 0)}{\left(s \mp \imath \omega_{\vec{k}}\right)\left[s^{2}+\omega_{\vec{q}}^{2}+s^{2} \tilde{\chi}_{e}(s)-\omega_{\vec{q}}^{2} \tilde{\chi}_{m}(s)\right]} \vec{e}_{-\vec{q} \lambda}\right\}
\end{aligned}
$$

where the upper (lower) sign corresponds to $\underline{\vec{A}}_{f}(\vec{q}, s)\left(\underline{\vec{A}}_{b}(\vec{q}, s)\right)$ respectively. Now taking the inverse Laplace transformation of $\underline{\vec{A}}_{f}(\vec{q}, s)$ and $\underline{A}_{b}(\vec{q}, s)$, we 
obtain a complete solution for $\vec{A}(\vec{r}, t)$

$$
\begin{aligned}
\vec{A}(\vec{r}, t) & =\sum_{\lambda=1}^{2} \int d^{3} \vec{q} \sqrt{\frac{\hbar}{2(2 \pi)^{3} \varepsilon_{0} \omega_{\vec{q}}}}\left[Z_{ \pm}\left(\omega_{\vec{q}}, t\right) e^{\imath \vec{q} \cdot \vec{r}} a_{\vec{q} \lambda}(0)+H . C .\right] \vec{e}_{\vec{q} \lambda} \\
& \pm \frac{1}{\varepsilon_{0}} \sum_{\lambda=1}^{2} \int \frac{d^{3} \vec{q}}{\sqrt{(2 \pi)^{3}}} \int d^{3} \vec{k}\left[\zeta_{ \pm}\left(\omega_{\vec{k}}, \omega_{\vec{q}}, t\right) d_{\lambda}(\vec{k}, \vec{q}, 0) e^{\imath \vec{q} \cdot \vec{r}}+H . C .\right] \vec{e}_{\vec{q} \lambda} \\
& +\sqrt{\frac{\mu_{0}}{\varepsilon_{0}}} \sum_{\lambda=1}^{2} \int \frac{d^{3} \vec{q} \omega_{\vec{q}}}{\sqrt{(2 \pi)^{3}}} \int d^{3} \vec{k}\left[\eta_{ \pm}\left(\omega_{\vec{k}}, \omega_{\vec{q}}, t\right) b_{\lambda}(\vec{k}, \vec{q}, 0) e^{\imath \vec{q} \cdot \vec{r}}+H . C .\right] \vec{e}_{\vec{q} \lambda},
\end{aligned}
$$

where the upper (lower) sign corresponds to $t>0(t<0)$. The functions $Z_{+}\left(\omega_{\vec{q}}, t\right), Z_{-}\left(\omega_{\vec{q}},-t\right), \zeta_{+}\left(\omega_{\vec{k}}, \omega_{\vec{q}}, t\right), \zeta_{-}\left(\omega_{\vec{k}}, \omega_{\vec{q}},-t\right), \eta_{+}\left(\omega_{\vec{k}}, \omega_{\vec{q}}, t\right), \eta_{-}\left(\omega_{\vec{k}}, \omega_{\vec{q}},-t\right)$ are given by

$$
\begin{aligned}
& Z_{ \pm}\left(\omega_{\vec{q}}, \pm t\right)=L^{-1}\left\{\frac{\left[s+s \tilde{\chi}_{e}(s) \mp \imath \omega_{\vec{q}}\right]}{s^{2}+\omega_{\vec{q}}^{2}+s^{2} \tilde{\chi}_{e}(s)-\omega_{\vec{q}}^{2} \tilde{\chi}_{m}(s)}\right\} \\
& \zeta_{ \pm}\left(\omega_{\vec{k}}, \omega_{\vec{q}}, \pm t\right)=f\left(\omega_{\vec{k}}\right) L^{-1}\left\{\frac{s}{\left(s \pm \imath \omega_{\vec{k}}\right)\left[s^{2}+\omega_{\vec{q}}^{2}+s^{2} \tilde{\chi}_{e}(s)-\omega_{\vec{q}}^{2} \tilde{\chi}_{m}(s)\right]}\right\} \\
& \eta_{ \pm}\left(\omega_{\vec{k}}, \omega_{\vec{q}}, \pm t\right)=g\left(\omega_{\vec{k}}\right) L^{-1}\left\{\frac{1}{\left(s \pm \imath \omega_{\vec{k}}\right)\left[s^{2}+\omega_{\vec{q}}^{2}+s^{2} \tilde{\chi}_{e}(s)-\omega_{\vec{q}}^{2} \tilde{\chi}_{m}(s)\right]}\right\}
\end{aligned}
$$

for $t>0$ and $L^{-1} f(s)$ is the inverse Laplace transformation of function $f(s)$. The transverse component of the electric field can be obtained from $\vec{E}^{\perp}=$ $-\frac{\partial \vec{A}}{\partial t}$. Having the vector potential $\vec{A}$, the transverse component of electric polarization density $\left(\vec{P}^{\perp}\right)$ and also magnetic polarization density $(\vec{M})$, can be obtained easily from relations (30) and (39).

Taking the Laplace transformation of the constitutive equation (30), the longitudinal component of the electric field can be written as

$$
\begin{aligned}
\vec{E}^{\|}(\vec{r}, t) & =-\frac{\vec{P} \|}{\varepsilon_{0}} \\
& =-\frac{1}{\varepsilon_{0}} \int \frac{d^{3} \vec{q}}{\sqrt{(2 \pi)^{3}}} \int d^{3} \vec{k}\left[Q_{ \pm}\left(\omega_{\vec{k}}, t\right) f\left(\omega_{\vec{k}}\right) d_{3}(\vec{k}, \vec{q}, 0) e^{i \vec{q} \cdot \vec{r}}+\text { H.C. }\right] \hat{q}
\end{aligned}
$$


where $Q_{+}\left(\omega_{\vec{k}}, t\right), Q_{-}\left(\omega_{\vec{k}},-t\right)$ are given by

$$
Q_{ \pm}\left(\omega_{\vec{k}}, \pm t\right)=L^{-1}\left\{\frac{1}{\left(1+\tilde{\chi}_{e}(s)\right)\left(s \pm i \omega_{\vec{k}}\right)}\right\},
$$

for $t>0$. In the following we consider some important examples.

\section{Example 1:}

Let $f\left(\omega_{\vec{k}}\right)=g\left(\omega_{\vec{k}}\right)=0$, then from (37) and (40), we have $\chi_{e}(t)=\chi_{m}(t)=0$, and from (151), we find

$$
Z_{+}\left(\omega_{\vec{q}}, t\right)=Z_{-}\left(\omega_{\vec{q}}, t\right)=e^{-i \omega_{\vec{q}} t}, \quad \eta_{ \pm}=\zeta_{ \pm}=0,
$$

therefore in this limiting case, quantization of electromagnetic field reduce to the usual quantization in the vacuum as expected.

\section{Example 2 :}

Take $\chi_{e}(t)$ and $\chi_{m}(t)$ as follows

$$
\begin{gathered}
\chi_{e}(t)= \begin{cases}\frac{\chi_{e}^{0}}{\triangle} & 0<t<\triangle, \\
0 & \text { otherwise, }\end{cases} \\
\chi_{m}(t)= \begin{cases}\frac{\chi_{m}^{0}}{\left(\chi_{m}^{0}+1\right) \triangle} & 0<t<\Delta \\
0 & \text { otherwise }\end{cases}
\end{gathered}
$$

where $\chi_{e}^{0}, \chi_{m}^{0}$ and $\triangle$ are some positive constants, using (38) and (41), we find the corresponding coupling functions as

$$
\begin{aligned}
\left|f\left(\omega_{\vec{k}}\right)\right|^{2} & =\frac{\hbar c^{3} \varepsilon_{0} \chi_{e}^{0}}{4 \pi^{2} \omega_{\vec{k}}^{2}} \frac{\sin ^{2} \frac{\omega_{\vec{k}}}{2}}{\frac{\omega_{\vec{k}}}{2}}, \\
\left|g\left(\omega_{\vec{k}}\right)\right|^{2} & =\frac{\hbar c^{3} \chi_{m}^{0}}{4 \pi^{2} \mu_{0}\left(\chi_{m}^{0}+1\right) \omega_{\vec{k}}^{2}} \frac{\sin ^{2} \frac{\omega_{\vec{k}} \triangle}{2}}{\frac{\omega_{\vec{k}}}{2}},
\end{aligned}
$$

and from (30) and (39) we have

$$
\begin{aligned}
& \vec{P}(\vec{r}, t)=\vec{P}_{N}(\vec{r}, t)+\frac{\varepsilon_{0} \chi_{e}^{0}}{\triangle} \int_{|t|-\triangle}^{|t|} d t^{\prime} \vec{E}\left(\vec{r}, \pm t^{\prime}\right) \\
& \vec{M}(\vec{r}, t)=\vec{M}_{N}(\vec{r}, t)+\frac{\chi_{m}^{0}}{\mu_{0}\left(\chi_{m}^{0}+1\right) \triangle} \int_{|t|-\triangle}^{|t|} d t^{\prime} \vec{B}\left(\vec{r}, \pm t^{\prime}\right),
\end{aligned}
$$


where $\vec{P}_{N}(\vec{r}, t)$ and $\vec{M}_{N}(\vec{r}, t)$ are the noise polarization densities (32) and (42) corresponding to the coupling functions obtained in (56).

In the limit $\triangle \rightarrow 0$, the coupling functions (56) and the noise polarization densities, tend to zero, and the relations (57) are reduced to

$$
\begin{aligned}
\vec{P}(\vec{r}, t) & =\varepsilon_{0} \chi_{e}^{0} \vec{E}(\vec{r}, t), \\
\vec{M}(\vec{r}, t) & =\frac{\chi_{m}^{0}}{\mu_{0}\left(\chi_{m}^{0}+1\right)} \vec{B}(\vec{r}, t) .
\end{aligned}
$$

In this limit, the electric field and the polarization densities are purely transverse and

$$
\begin{aligned}
& Z_{-}\left(\omega_{\vec{q}}, t\right)=Z_{+}\left(\omega_{\vec{q}}, t\right)=\cos \tilde{\omega}_{\vec{q}} t-i \sqrt{\frac{1+\chi_{m}^{0}}{1+\chi_{e}^{0}}} \sin \tilde{\omega}_{\vec{q}} t, \\
& \tilde{\omega}_{\vec{q}}=\frac{\omega_{\vec{q}}}{\sqrt{\left(1+\chi_{e}^{0}\right)\left(1+\chi_{m}^{0}\right)}}, \\
& \eta_{ \pm}\left(\omega_{\vec{q}}, t\right)=\zeta_{ \pm}\left(\omega_{\vec{q}}, t\right)=0 .
\end{aligned}
$$

Also the electromagnetic energy inside the dielectric is

$$
\begin{aligned}
\int\left[\frac{1}{2} \vec{E} \cdot \vec{D}+\frac{1}{2} \vec{H} \cdot \vec{B}\right] d^{3} r & =\int d^{3} \vec{q}\left[\frac{1}{2 \varepsilon_{0}\left(1+\chi_{e}^{0}\right)} \vec{D}(\vec{q} \cdot 0) \cdot \underline{D}^{\dagger}(\vec{q} \cdot 0)\right. \\
& \left.+\frac{\varepsilon_{0} \omega_{\vec{q}}^{2}}{2\left(1+\chi_{m}^{0}\right)} \underline{\vec{A}}(\vec{q}, 0) \cdot \underline{\vec{A}}^{\dagger}(\vec{q}, 0)\right]
\end{aligned}
$$

where $\underline{D}$ is the Fourier transform of the displacement field. The energy given by (60) is a constant of motion contrary to the vacuum expression $\int\left[\frac{1}{2} \varepsilon_{0} \vec{E}^{2}+\frac{\vec{B}^{2}}{2 \mu_{0}}\right] d^{3} r$, which is not clearly a constant of motion. This example shows that this model can be applied to a nondispersive magneto-dielectric medium.

Example 3: Let $\chi_{e}(t)=\beta u(t)$ and $\chi_{m}(t)=0$, where $u(t)$ is the step function

$$
u(t)= \begin{cases}1 & t>0 \\ 0 & t \leq 0\end{cases}
$$


and $\beta$ is a positive constant, then using (38) and (41), we find

$$
\left|f\left(\omega_{\vec{k}}\right)\right|^{2}=\frac{\hbar c^{3} \varepsilon_{0} \beta}{4 \pi^{2} \omega_{\vec{k}}^{3}}, \quad g\left(\omega_{\vec{k}}\right)=0,
$$

and accordingly we can rewrite (46) as

$$
\begin{aligned}
\underline{\ddot{\vec{A}}}+\omega_{\vec{q}}^{2} \underline{\vec{A}}+\beta \underline{\dot{\vec{A}}} & =-\imath \sqrt{\frac{\hbar c^{3} \beta}{4 \pi^{2} \varepsilon_{0}}} \int \frac{d^{3} \vec{k}}{\sqrt{(2 \pi)^{3} \omega_{\vec{k}}}} \sum_{\lambda=1}^{2}\left[d_{\lambda}(\vec{k}, \vec{q}, 0) e^{-\imath \omega_{\vec{k}} t} \vec{e}_{\vec{q} \lambda}\right. \\
& \left.-d_{\lambda}^{\dagger}(\vec{k},-\vec{q}, 0) e^{\imath \omega_{\vec{k}} t} \vec{e}_{-\vec{q} \lambda}\right]
\end{aligned}
$$

which has a dissipative term proportional to the first time derivative of dynamical variable $\underline{\vec{A}}$. From (151), one can obtain

$$
\begin{aligned}
& Z_{ \pm}\left(\omega_{\vec{q}}, t\right)=e^{\mp \frac{\beta}{2} t}\left[ \pm \frac{\beta}{2 \Omega_{\vec{q}}} \sin \Omega_{\vec{q}} t+\cos \Omega_{\vec{q}} t-\frac{i \omega_{\vec{q}}}{\Omega_{\vec{q}}} \sin \Omega_{\vec{q}} t\right], \\
& \zeta_{ \pm}\left(\omega_{\vec{k}}, \omega_{\vec{q}}, t\right)=\sqrt{\frac{\hbar c^{3} \beta \varepsilon_{0}}{4 \pi^{2} \omega_{\vec{k}}^{3}}}\left\{\mp \frac{i \omega_{\vec{k}} e^{-i \omega_{\vec{k}} t}}{\omega_{\vec{q}}^{2}-\omega_{\vec{k}}^{2} \mp i \beta \omega_{\vec{k}}}+\right. \\
& \left.e^{\mp \frac{\beta}{2} t}\left[\frac{\left(-\frac{\beta}{2}+i \Omega_{\vec{q}}\right) e^{ \pm i \Omega_{\vec{q}} t}}{2 i \Omega_{\vec{q}}\left(-\frac{\beta}{2}+i \Omega_{\vec{q}} \pm i \omega_{\vec{k}}\right)}+\frac{\left(\frac{\beta}{2}+i \Omega_{\vec{q}}\right) e^{\mp i \Omega_{\vec{q}} t}}{2 i \Omega_{\vec{q}}\left(-\frac{\beta}{2}-i \Omega_{\vec{q}} \pm i \omega_{\vec{k}}\right)}\right]\right\},
\end{aligned}
$$

where $\Omega_{\vec{q}}=\sqrt{\omega_{\vec{q}}^{2}-\frac{\beta^{2}}{4}}$. The asymptotic solution of $\vec{A}(\vec{r}, t)$ in large-time limit is

$$
\vec{A}(\vec{r}, t)=\mp \imath \sqrt{\frac{\hbar c^{3} \beta}{4 \pi^{2} \varepsilon_{0}}} \sum_{\lambda=1}^{2} \int \frac{d^{3} \vec{q}}{\sqrt{(2 \pi)^{3}}} \int \frac{d^{3} \vec{k}}{\sqrt{\omega_{\vec{k}}}}\left[\frac{d_{\lambda}(\vec{k}, \vec{q}, 0) e^{-\imath \omega_{\vec{k}} t+i \vec{q} \cdot \vec{r}}}{\omega_{\vec{q}}^{2}-\omega_{\vec{k}}^{2} \mp \imath \beta \omega_{\vec{k}}}-\text { H.C. }\right] .
$$

Using (52), it is easy to show that the longitudinal component of the electric field in the limit $t \rightarrow \pm \infty$ is

$$
\begin{aligned}
\vec{E}^{\|}(\vec{r}, t) & =-\frac{\vec{P} \|}{\varepsilon_{0}} \\
& = \pm i \sqrt{\frac{\hbar c^{3} \beta}{4 \pi^{2} \varepsilon_{0}}} \int \frac{d^{3} \vec{q}}{\sqrt{(2 \pi)^{3}}} \int \frac{d^{3} \vec{k}}{\sqrt{\omega_{\vec{k}}}}\left[\frac{d_{3}(\vec{k}, \vec{q}, 0)}{\beta \mp i \omega_{\vec{k}}} e^{-i \omega_{\vec{k}} t+i \vec{q} \cdot \vec{r}}-H . C .\right] .
\end{aligned}
$$




\section{Example 4: A simple model for $\tilde{\chi}_{e}(s)$}

If we neglect the difference between local and macroscopic electric field for substances with a low density, then the classical equation of a bound atomic electron in an external electric field is

$$
\ddot{\vec{r}}+\gamma \dot{\vec{r}}+\omega_{0}^{2} \vec{r}=-\frac{e}{m} \vec{E}(t),
$$

where the influence of the magnetic force has been neglected compared to the electric force. The parameter $\gamma$ is a damping coefficient and the force exerted on the electron due to atom is taken to be simply a spring force with

frequency $\omega_{0}$. If $\tilde{\vec{E}}(s)$ and $\tilde{\vec{r}}(s)$ are the Laplace transformations of $\vec{E}(t)$ and $\vec{r}(t)$ respectively, then from (67) we can find

$$
\tilde{\vec{r}}(s)=\frac{-\frac{e}{m} \tilde{\vec{E}}(s)}{s^{2}+\gamma s+\omega_{0}^{2}} .
$$

Now let there be $N$ molecules per unit volume with $z$ electrons per molecule such that $f_{j}$ electrons of any molecule have a bound frequency $\omega_{j}$ and a damping coefficient $\gamma_{j}$. The Laplace transformation of the polarization density is

$$
\tilde{\vec{P}}(s)=\frac{N e^{2}}{m} \sum_{j} \frac{f_{j}}{s^{2}+\gamma_{j} s+\omega_{j}^{2}} \tilde{\vec{E}}(s) .
$$

If $\omega_{j}$ and $\gamma_{j}$ are identical for all of electrons, then from (69) we can write

$$
\begin{array}{ll}
\tilde{\chi}_{e}(s)=\frac{\omega_{p}^{2}}{s^{2}+\gamma s+\omega_{0}^{2}}, & \omega_{p}^{2}=\frac{N e^{2} z}{m \varepsilon_{0}}, \\
\chi_{e}(t)=\omega_{p}^{2} e^{-\frac{\gamma t}{2}} \frac{\sin \nu_{0} t}{\nu_{0}} u(t), & \nu_{0}^{2}=\omega_{0}^{2}-\frac{\gamma^{2}}{4},
\end{array}
$$

where $u(t)$ is the step function defined in (61). We can obtain the coupling function $f\left(\omega_{\vec{k}}\right)$ from (38) as

$$
\left|f\left(\omega_{\vec{k}}\right)\right|^{2}=\frac{\hbar c^{3} \varepsilon_{0} \omega_{p}^{2}}{16 \pi^{2} \nu_{0} \omega_{\vec{k}}^{2}}\left\{\frac{\gamma}{\frac{\gamma^{2}}{4}+\left(\nu_{0}-\omega_{\vec{k}}\right)^{2}}-\frac{\gamma}{\frac{\gamma^{2}}{4}+\left(\nu_{0}+\omega_{\vec{k}}\right)^{2}}\right\} .
$$

If $\gamma=0$, then the dielectric substance is a nondissipative one and the coupling function takes the form

$$
\left|f\left(\omega_{\vec{k}}\right)\right|^{2}=\frac{\hbar c^{3} \varepsilon_{0} \omega_{p}^{2}}{8 \pi \nu_{0}^{3}} \delta\left(\nu_{0}-\omega_{\vec{k}}\right) .
$$


In this case, the noise electric polarization density is nonzero only for the resonant frequency $\left(\omega=\omega_{0}\right)$ of the equation $\ddot{\vec{r}}+\omega_{0}^{2} \vec{r}=-\frac{e}{m} \vec{E}_{0} e^{-i \omega_{0} t}$. In the resonant case, energy of electromagnetic field will be absorbed by the medium. By using (51), we can obtain $Z_{+}\left(\omega_{\vec{q}}, t\right)$ and $Z_{-}\left(\omega_{\vec{q}},-t\right)$, as

$$
Z_{ \pm}\left(\omega_{\vec{q}}, \pm t\right)=L^{-1}\left\{\frac{\left(s \mp i \omega_{\vec{q}}\right)\left(s^{2}+\omega_{0}^{2}\right)+s \omega_{p}^{2}}{s^{4}+s^{2}\left(\omega_{0}^{2}+\omega_{\vec{q}}^{2}+\omega_{p}^{2}\right)+\omega_{\vec{q}}^{2} \omega_{0}^{2}}\right\}
$$

for $t>0$. The equation (173) can be solved by calculating the residues of the function

$$
\frac{\left[\left(s \mp i \omega_{\vec{q}}\right)\left(s^{2}+\omega_{0}^{2}\right)+s \omega_{p}^{2}\right] e^{ \pm s t}}{s^{4}+s^{2}\left(\omega_{0}^{2}+\omega_{\vec{q}}^{2}+\omega_{p}^{2}\right)+\omega_{\vec{q}}^{2} \omega_{0}^{2}},
$$

by extending $\mathrm{s}$ to the domain of complex variables. Similarly $\zeta_{ \pm}$can be obtained from (151) with $f\left(\omega_{\vec{k}}\right)$ given by (72). In this case we have

$$
\begin{aligned}
Q_{ \pm}\left(\omega_{\vec{k}}, t\right) & =\frac{\omega_{0}^{2}-\omega_{\vec{k}}^{2}}{\omega_{0}^{2}+\omega_{p}^{2}-\omega_{\vec{k}}^{2}} e^{-i \omega_{\vec{k}} t} \\
& +\frac{\omega_{p}^{2}}{2 \sqrt{\omega_{0}^{2}+\omega_{p}^{2}}}\left\{\frac{e^{ \pm i \sqrt{\omega_{0}^{2}+\omega_{p}^{2}} t}}{\sqrt{\omega_{0}^{2}+\omega_{p}^{2}} \pm \omega_{\vec{k}}}+\frac{e^{\mp i \sqrt{\omega_{0}^{2}+\omega_{p}^{2}} t}}{\sqrt{\omega_{0}^{2}+\omega_{p}^{2}} \mp \omega_{\vec{k}}}\right\}
\end{aligned}
$$

The longitudinal component of the electric field can be obtained from (52), using $f\left(\omega_{\vec{k}}\right)$ and $Q_{ \pm}\left(\omega_{\vec{k}}, t\right)$ given by (72) and (75) respectively.

If $\gamma \neq 0$, the substance is of dissipative kind and $\vec{A}(\vec{r}, t)$, can be obtained from (150) with $f\left(\omega_{\vec{k}}\right)$ given by (71) and $\tilde{\chi}_{e}(s)$ given by (70). In this case the longitudinal component of the electric field can be obtained from (52). This example shows that this model of quantization of the electromagnetic field is applicable to both dissipative and nondissipative dielectrics.

\section{Concluding remarks}

By modeling a linear, polarizable and magnetizable medium with two quantum fields E and M, electromagnetic field is quantized in the medium, consistently and systematically. There are many models for the environment with the same electric and magnetic properties. In other words, there are many dispersion relations and coupling functions leading to the same suceptibilities of the medium. Therefore one can take the simplest physical dispersion 
relation. Once a dispersion relation is defined, then for any definite magnetodielectric medium, i.e., $\chi_{e}(t)$ and $\chi_{m}(t)$ are known functions, one can find the corresponding coupling functions $f\left(\omega_{\vec{k}}, \vec{r}\right)$ and $g\left(\omega_{\vec{k}}, \vec{r}\right)$. The coupling functions describe the electric and magnetic properties of the medium macroscopically. These functions also couple electromagnetic field to the medium through the quantum fields $\mathrm{E}$ and $\mathrm{M}$. The explicit forms of the noise densities are derived. Since the coupling functions are common factors in the noise densities and susceptibilities, the relation between the strengths of the noise densities and susceptibilities is clear. In this approach, both Maxwell and constitutive equations are obtained as Heisenberg equations of motion. In the limiting case, i.e., when there is no medium, the approach tends to the usual quantization of the electromagnetic field in vacuum as expected. This model of quantization is applicable to both dispersive and nondispersive magneto-dielectrics.

\section{References}

[1] R. J. Glauber, M. Lewenstein, Phys. Rev. A 43, 467 (1991)

[2] W. Vogel, D. G. Welsch, S. Wallentowitz, Quantum optics an introduction, Wiley-Vch(2001)

[3] D. Kumar, Pramana-J. phys, Vol. 59, No. 2 (2002)

[4] Z. Lenac, Phys, Rev. A 68, 063815 (2003)

[5] B. Huttner, S. Barnett, Phys. Rev. A 46, 4306 (1992)

[6] T. Gruner, D. G. Welsch, Phys. Rev. A 53, 1818 (1996)

[7] L. G. Suttorp, M. Wubs, Phys. Rev. A 70, 013816(2004)

[8] G. W. Ford, J. T. Lewis, R. F. O'Connel, Phys. Rev. A 37, 4419 (1987)

[9] G. W. Ford, J. T. Lewis, R. F. O’Connel, Phys. Rev. Lett 55, 2273 (1985)

[10] R. Matloob, Phys. Rev. A 70, 022108 (2004)

[11] S. Savasta, O. Di Stefano, R. Girlanda, Phys. Rev. A 65,043801 (2002) 
[12] O. Di Stefano, S. Savasta, R. Girlanda, J. Opt. B: Quantum semiclass, Opt. 3, 288 (2001)

[13] H. T. Dung, S. Y. Buhmann, L. Knöll, D. G. Welsch, Phys. Rev. A $68,043816(2003)$

[14] R. Matloob, R. Loudon, Phys. Rev. A 60, 3421 (1999)

[15] M. Khanbekyan, L. Knoll, D. G. Welsch, Phys. Rev. A 67, 063812 (2003)

[16] S. Scheel, D. G. Welsch, quant-ph/9906001 V2 (2004)

[17] J. C. Garrison, R. Y. Chiao, Phys. Rev. A 70, 053826 (2004)

[18] M. Artoni, R. Loudon, Phys. Rev. A 59 (1999)

[19] H. Khosravi, R. Loudon, Proc. R. Soc. London, Ser. A 436, 373 (1992)

[20] S. Barnett, B. Huttner, R. Loudon, Phys. Rev. Lett. 68, 3698 (1992)

[21] S. Barnett, B. Huttner, R. Matloob, R. Loudon, J. Phys. B 29, 3763 (1996)

[22] A. O. Caldeira, A. J. Leggett, Phys. Rev. Lett. 46, 211 (1981)

[23] A. O. Caldeira, A. J. Leggett, Ann. Phys. (N.Y.) 149, 374 (1983)

[24] R. Matloob, Optics Communications 192,287-297(2001) 